\title{
HÁBITOS E CONSUMO AUMENTARES DE ATLETAS NADADORES
}

\author{
FEEDING HABTS AND INTAKES OF SWMMER ATHLEIES
}

lleana Mourão KAZAPI ${ }^{1}$

Lúcia Andréa Zanete RAMOS

\begin{abstract}
RESUM 0
Estudou-se 27 atletas nadadores (14 mulheres e 13 homens) com idade entre 13 e 21 anos, com o objetivo de conhecer seus hábitos alimentares e consumo alimentar, aplicando-se questionários de registro e freqüencia alimentar. O percentual de adequação foi determinado comparando-se as necessidades de cada indivíduo (calculadas teoricamente) com as quantidades consumidas. Os resultados indicaram que o consumo energético foi maior para os homens (média de $3125 \mathrm{Kcal}$ contra $1865 \mathrm{Kcal}$ das mulheres). O consumo dos demais nutrientes também apresentou-se superior para o sexo masculino, exceto para a vitamina C. A contribuição percentual de macronutrientes apresentou-se desequilibrada tanto para os homens quanto para as mulheres. Os homens apresentaram uma adequação baixa (70-80\%) para os carboidratos e vitamina A, uma adequação excedente (> 110\%) para proteínas, gordura, vitamina C e ferro, e uma adequação normal para os demais nutrientes. As mulheres apresentaram uma adequação marginal ( $<70 \%)$ para os carboidratos, energia e cálcio; excedente para a vitamina $C$ e normal para os demais nutrientes.
\end{abstract}

Termos de indexação: Consumo de alimentos, hábitos alimentares, nutrição, dieta, atletas, nadadores.

\begin{abstract}
Twenty-seven swimmer athletes (14 women and 13 men), aged 13 to 21 years old, were studied to determine their dietary habits and food intakes. The subjects recorded their food intake and frequency. The percentage of their nutritional adequacy was determined comparing the nutritional requirements of each person (theoretically calculated) with the consumed quantities. The results indicated that the caloric intake was higher for men (3124,7 Kcal against $1865 \mathrm{Kcal}$ for women). The intake of all the other nutrients was observed to be higher for men, except for vitamin $C$. The diet was unbalanced (macronutrients) for both sexes. Men presented a low adequacy (70-80\%) of carbohydrates and vitamin A, excess of protein, fat, vitamin C and iron (> 110\%), and a normal adequacy
\end{abstract}

\footnotetext{
(1) Nutricionista, Professora Adjunta da área de Nutrição Básica do Departamento de Nutrição da Universidade Federal de Santa Catarina, Campus Universitário Trindade, Centro de Ciências da Saúde, Caixa Postal 476, 88040-900 Florianópolis, SC.

(2) Nutricionista, Professora Assistente da área de Nutrição Clínica do Departamento de Nutrição da Universidade Federal de Santa Catarina.
} 
of the other nutrients. Women presented a marginal adequacy of carbohydrates, energy and calcium ( $<70 \%)$, excess of vitamin $C$, and a normal adequacy of the other nutrients.

Index terms: food consumption, food habits, nutrition, diet, athletes, swimmers.

\section{INTRO DUÇÃO}

Existe em Nutrição muitas crenças e concepções quanto ao desempenho atlético. Por exemplo, em épocas passadas o consumo exagerado de carne era associado com a formação de músculos e a geração de força. Esta crença continua a vigorar até hoje entre atletas e treinadores por acreditarem que alimentos ricos em proteína animal teriam o poder de aumentar o desempenho em esportes de força e resistência (VAN ERP-BAART et al., 1989).

A maior parte da população acredita que atletas são os modelos de saúde física e mental, e por isto seu estado nutricional e o seu consumo alimentar devam ser superiores ao do não atleta. Contudo inquéritos nutricionais têm mostrado que as medidas antropométricas e o consumo alimentar de atletas são muito similares às médias populacionais, exceto para energia (SHORT \& SHORT, 1983).

De acordo com BROTHERHOOD (1984) "poucos atletas seguem o melhor padrão dietético para o esporte; eles ingerem muito mais gordura e proteína em detrimento dos carboidratos".

Geralmente os atletas não consomem quantidades adequadas de carboidratos e fazem uso de altas concentrações de carboidratos refinados ou gorduras quando suas necessidades energéticas estão muito aumentadas e muitos fazem uso de suplementos vitamínicos e de minerais (LEAF, 1989; LEAF \& FRISA, 1989, 1989).

No que se refere a ingestão de proteínas, existem controvérsias sobre o assunto entre diferentes autores. Existem por um lado, aqueles que acham que deve haver um aumento da ingestão em até quatro vezes mais que a recomendação, alegando que ocorrem perdas de aminoácidos durante o treinamento e competições. Por outro lado, encontram-se aqueles que defendem uma ingestão protéica de 11 a 14\%, mostrando que atletas conseguem um balanço nitrogenado positivo mesmo durante o treinamento pesado (CELLEJOWA \& HOMA, 1970; LEMON et al, 1984; BURKE \& READ, 1987; LEMON, 1987).
De acordo com os dados disponíveis na literatura, parece que existem poucas razões para que a dieta dos atletas seja diferente das pessoas sedentárias. Só existe exceção no que se refere ao aporte energético, que deve ser maior, e a ingestão de carboidratos que deve vir em maior quantidade na forma de carboidratos complexos (BUSKIRK, 1991).

BROTHERHOOD (1984), fez um compêndio da ingestão energética e da contribuição percentual de macronutrientes de atletas e não atletas. O estudo revelou que os atletas consumiam mais energia, mas no que se refere a contribuição percentual de macronutrientes, não ocorreu diferenças significativas entre os dois grupos, os carboidratos contribuíam com 40 a 50\% da energia, os lipídeos com 31 a 41\% e as proteínas com 11 a $17 \%$.

A ingestão de energia e nutrientes varia de esporte para esporte; ginastas geralmente têm a menor ingestão calórica e maratonistas e triatletas a maior. Entretanto, é interessante citar que a contribuição percentual de macronutrientes para qualquer esporte é desequilibrada (BROTHERHOOD, 1984).

A nutrição parece ser ótima e adequada para o desempenho atlético quando quantidades de alimentos são suficientes para satisfazer o aumento das necessidades durante o treinamento e a competição. Uma dieta balanceada, que contenha 12 a $15 \%$ de proteínas, 20 a $30 \%$ de lipídeos e 55 a $65 \%$ de carboidratos poderia atender as necessidades dos atletas (LEAF \& FRISA, 1989).

Uma ingestão inadequada de vitaminas e minerais prejudica e muitas vezes impede o desempenho físico. O exercício físico pode aumentar a necessidade de certas vitaminas e minerais envolvidos em processos oxidativos. Contudo, com uma dieta balanceada alcança-se as necessidades desses micronutrientes (BELKO, 1987).

Numerosos estudos demonstram que a ingestão e a adequação marginal em atletas de resistência, para certos nutrientes entre os quais: ferro, zinco, cálcio, vitaminas $\mathrm{B}_{6}, \mathrm{~B}_{2}, \mathrm{~B}_{1}$, A e $\mathrm{C}$, principalmente quando acompanhadas de uma baixa ingestão energética 
prejudicam o desempenho (CLEMENT \& ASMUNDSON, 1982; BURKE \& READ, 1987; KHOO et al., 1987; HULTMAN, 1989; SHILS et al., 1995).

A influência do espaçamento e do número de refeições tem sido estudada em relação ao seu efeito sobre o desempenho. MAYER \& BULLEN (1960), encontraram que refeições freqüentes levam a altos níveis de desempenho, mostrando que o uso de cinco refeições aumenta a produção do trabalho físico.

A partir desta revisão, surgiu o interesse em realizar uma pesquisa com atletas nadadores com o objetivo de conhecer seus hábitos e consumo alimentares, bem como avaliar a adequação do consumido com o recomendado.

\section{MATERIALE MÉTODOS}

\section{Casuística}

Foram estudados 27 atletas nadadores, sendo 14 do sexo feminino e 13 do sexo masculino, com idade compreendida entre 13 e 21 anos, pertencentes ao Lira Tênis Clube e que foram voluntários para este trabalho.

Esses atletas tinham um treino médio diário de 2 horas, que consistia nos quatro estilos de natação (crawl, peito, costas egolfinho). Eles foram pesquisados durante a fase de treinamento, de abril a dezembro de 1994. Nessa fase os atletas desenvolviam um trabalho que variava entre 75 a 90 do $\mathrm{VO}_{2}$ Máximo.

\section{Ava lia çã o do consumo dietétic 0}

Muito se tem escrito sobre as vantagens e desvantagens dos inquéritos dietéticos baseados no uso dos métodos do recordatório de 24 horas e do registro alimentar que podem variar de um a sete dias. Entretanto a maioria dos autores consideram o método do registro, o melhor de ser aplicado e o que melhor retorno traz, no que se refere ao consumo, pois o uso de múltiplos dias, permitequantificara ingestãohabitual de macronutrientes e de energia do indivíduo ou do grupo (GERSOVITZ et al., 1978; KRALL \& DWYER, 1987).

As informações sobre o consumo dietético dos atletas foram coletadas através da aplicação do método do registro alimentar de sete dias, no qual os indivíduos registravam todas as refeições, alimentos e preparações, em medidas caseiras que depois eram convertidas em gramas a fim de se calcular o consumo de energia e nutrientes, através de tabela de composição de alimentos (INSTITUTO..., 1979).

Aplicou-se também, um questionário de freqüência qualitativa de alimentos, no qual se obteve informações sobre o número de vezes que cada alimento foi consumido no período de sete dias, a fim de se conhecer os hábitos alimentares dos atletas. Os alimentos foram tabulados de acordo com a freqüência que apareciam e em grupos alimentares.

\section{Cálc ulo da srecomenda çõesnutric iona is}

Para o cálculo das recomendações nutricionais, aplicou-se um questionário onde foram registradas todas as atividades realizadas pelos indivíduos, horas gastas nas respectivas atividades e data de nascimento.

A partir dessas informações e dos dados antropométricos de peso e altura, calculou-se a recomendação de energia pelo método dos múltiplos do metabolismo basal (ORGANIZACIÓN..., 1985).

Em relação à contribuição percentual de carboidratos (CHO), proteínas (PTN) e lipídeos (LIP), a recomendação baseou-se na Sociedade Brasileira de Alimentação e Nutrição (VANNUCCHI et al., 1990). As recomendações de vitaminas e minerais foram calculadas pela Recommended Dietary Allowances (MONSEN, 1989).

Através do cálculo da recomendação e do consumo, verificou-se a adequação do nutriente em questão, em termos percentuais do quanto o nutriente preencheria as recomendações, através da fórmula :

Adequação $(\%)=($ Consumido $/$ recomendado $)$ $\mathrm{x} 100$

Assumiu-se os seguintes valores para a adequação :

Excedente: > 100 \% de adequação

Normal: 80 a 100\% de adequação

Baixo: 70 a 80 \% de adequação

Insuficiente ou marginal: $<70 \%$ de adequação 


\section{Análise esta tístic a}

Os dados foram apresentados como média e desvio padrão. Comparações foram feitas através do teste " $t$ " de Student, com um nível de significância de $5 \%$.

\section{RESULTADOS}

\section{Ingestão e adequação de energia e nutrientes}

Entre homens e mulheres, a ingestão dietética reflete a ampla variabilidade entre os indivíduos de sexos diferentes, sugerindo diferentes hábitos e regimes dietéticos. A ingestão de energia e de nutrientes (exceto vitamina $\mathrm{C}$ ) foi maior para os homens, diferença estatisticamente significativa. A média de ingestão de calorias para os homens foi de 3125 e para as mulheres de 1 865; esta diferença para o sexo feminino pode ser explicada pelo fato de que as mulheres citaram o medo de engordar, fazendo regimes totalmente desequilibrados. Em relação as proteínas, os homens tiveram um consumo de quase duas vezes maior do que as mulheres; e se relacionarmos em $\mathrm{g} / \mathrm{Kg}$ de peso, iremos encontrar uma ingestão de $2,2 \mathrm{~g} / \mathrm{Kg} /$ dia e $1,4 \mathrm{~g} / \mathrm{Kg} /$ dia para homens e mulheres respectivamente. Esta ingestão é maior do que a recomendação de $1,0 \mathrm{~g} / \mathrm{Kg} /$ dia encontrada por BUTTERFIELD \& CALLOWAY (1984) e por SOARES et al. (1994) e maior do que o recomendado por LEMON (1987). Este elevado consumo protéico, talvez seja explicado pela falsa crença de que este tipo de nutriente aumenta a força, o desempenho e a resistência (Tabela 1).

Tabela 1. Ingestão de energia e nutrientes baseada em registro alimentar de 7 dias, dos atletas nadadores de ambos os sexos (média e desvio-padrão) Florianópilis - SC, 1994.

\begin{tabular}{lrlrrrr}
\hline $\begin{array}{l}\text { Energia } \\
\text { Nutrientes }\end{array}$ & \multicolumn{3}{c}{ Homens $(\mathrm{n}=13)$} & \multicolumn{3}{c}{ Mulheres $(\mathrm{n}=14)$} \\
\hline Kcal & 3125 & \pm & $829^{*}$ & 1865 & \pm 662 \\
Proteína (g) & 141 & \pm & $36^{*}$ & 78 & \pm & 26 \\
Lipídeo (g) & 116 & \pm & $30^{*}$ & 85 & \pm & 40 \\
Carboidrato (g) & 361 & \pm & $109^{*}$ & 244 & \pm & 98 \\
Vitamina A (mcg) & 783 & \pm & $551^{*}$ & 720 & \pm 393 \\
Vitamina C (mg) & 102 & \pm & $77^{*}$ & 140 & \pm & 78 \\
Ferro (mg) & 24 & \pm & $10^{*}$ & 14 & \pm 6 \\
Cálcio(mg) & 1099 & \pm & $55^{*}$ & 697 & \pm & 32 \\
$*$ * $<0,05$ & & & & & &
\end{tabular}

Quando analisamos a contribuição percentual das proteínas, encontramos um percentual de 19,31\% e $17,0 \%$ para homens e mulheres respectivamente, diferença esta estatisticamente significativa. Esta contribuição percentual ultrapassa o que é recomendado para este nutriente que deve fornecer em torno de 12,0 a $15,0 \%$ da energia da dieta (HULTMAN et al., 1988; LEMON, 1994, WILLIAMS \& DEVLIN, 1994). Estes dados foram semelhantes aos encontrados por alguns autores onde este percentual teve uma variação de 18,6 a 37,0\% (CHEN, 1989; BAZARRE et al., 1990; FABER et al, 1990).

O consumo de gordura foi também bastante elevado e semelhante em ambos os sexos: $116 \mathrm{~g}$ para os homens e $85 \mathrm{~g}$ das mulheres, diferença esta estatisticamente significativa. O percentual de contribuição energético das gorduras ficou acima da recomendação, que é de $30 \%$, os homens com um resultado de $35 \%$ e as mulheres de $34 \%$ (BAR-OR \& UNNITHAN, 1994). Para os carboidratos (Tabela 2), ambos os sexos consomem abaixo do recomendado para atletas e mesmo para a população em geral, que varia conforme a modalidade esportiva entre 55,0 a $65,0 \%$ ou entre 60,0 a $70,0 \%$. Os resultados encontrados foram de $45,69 \%$ para os homens e de $49,0 \%$ para as mulheres (BLAIR et al.,1989; HARGREAVES, 1994).

Tabela 2. Contribuição percentual dos macronutrientes em relação a ingestãoenergética, dos nadadores de ambos os sexos (média e desvio padrão), Florianópolis-SC, 1994.

\begin{tabular}{lcc}
\hline Macronutrientes & Homens $(\mathrm{n}=13)$ & Mulheres $(\mathrm{n}=14)$ \\
\hline Lipídeo & $35,00 \pm 4,15^{*}$ & $34,0 \pm 8,5$ \\
Carboidrato & $45,69 \pm 6,68^{*}$ & $49,0 \pm 10,5$ \\
Proteína & $19,31 \pm 5,66^{*}$ & $17,0 \pm 3,9$ \\
\hline
\end{tabular}

Quando parte-se para a análise da adequação de energia e nutrientes, verifica-se que para os homens ocorreu uma baixa adequação (70 a 80\%) para os carboidratos e a vitamina A; uma adequação excedente (> 110\%) para proteína, lipídeos, vitamina C e ferro e uma adequação normal para energia e os demais nutrientes. Já para as mulheres foi verificado uma adequação marginal $(<70 \%)$ para energia, carboidratos e cálcio; uma adequação excedente para a vitamina $C$ e uma adequação normal para os demais nutrientes (Tabela 3) . 
Tabela 3. Percentual de adequação de energia e nutrientes de atletas nadadores de ambos os sexos. Florianópolis - SC, 1994.

\begin{tabular}{|c|c|c|c|c|c|c|}
\hline \multirow{2}{*}{ Nutriente } & \multicolumn{3}{|c|}{ Homens } & \multicolumn{3}{|c|}{ Mulheres } \\
\hline & Consumido & Recomendado & Adequação (\%) & Consumido & Recomendado & Adequação (\%) \\
\hline Kcal & 3125,0 & 3237,0 & $96,5^{*}$ & 1865,0 & 2708,0 & 69,0 \\
\hline PTN & $141,0 \mathrm{~g}$ & $104,0 \mathrm{~g}$ & $135,6^{*}$ & $78,0 \mathrm{~g}$ & $86,0 \mathrm{~g}$ & 91,0 \\
\hline LIP & $116,0 \mathrm{~g}$ & $97,0 \mathrm{~g}$ & $119,5^{*}$ & $80,5 \mathrm{~g}$ & $82,1 \mathrm{~g}$ & 98,0 \\
\hline $\mathrm{CHO}$ & $361,0 \mathrm{~g}$ & $484,0 \mathrm{~g}$ & $74,6^{*}$ & $244,0 \mathrm{~g}$ & $450,0 \mathrm{~g}$ & 54,0 \\
\hline Vitamina A & $783,0 \mathrm{mcg}$ & $1000,0 \mathrm{mg}$ & $78,3^{*}$ & $720,0 \mathrm{mcg}$ & $800,0 \mathrm{mcg}$ & 90,0 \\
\hline Vitamina C & $102,0 \mathrm{mg}$ & $60,0 \mathrm{mg}$ & $170,0^{*}$ & 139,7 mg & $60,0 \mathrm{mg}$ & 233,0 \\
\hline Ferro & 24,3 mg & $12,0 \mathrm{mg}$ & $202,5^{*}$ & $14,4 \mathrm{mg}$ & $15,0 \mathrm{mg}$ & 96,0 \\
\hline Cálcio & $1099,0 \mathrm{mg}$ & $1200,0 \mathrm{mg}$ & $92,0^{*}$ & $696,9 \mathrm{mg}$ & $1200,0 \mathrm{mg}$ & 58,0 \\
\hline
\end{tabular}

$* \mathrm{p}<0,05$

Tabela 4. Consumo diário dos grupos de alimentos pelos atletas nadadores de ambos os sexos. Florianópolis - SC, 1994.

\begin{tabular}{lcc}
\hline Grupo Alimentar & Homens & Mulheres \\
\cline { 2 - 2 } Pães e amido & 87 & \\
Gorduras & 65 & 80 \\
Açúcar e bebidas & 66 & 50 \\
Leite e derivados & 66 & 90 \\
Carnes & 80 & 70 \\
Vegetais e frutas & 30 & 25 \\
\hline
\end{tabular}

Tabela 5. Refeições ingeridas pelos atletas nadadores de ambos os sexos. Florianópolis - SC, 1994.

\begin{tabular}{lcc}
\hline Refeições & Homens & Mulheres \\
\cline { 2 - 3 } Desjejum & & \\
Colação & 80 & 80 \\
Almoço & 87 & 80 \\
Jantar & 100 & 100 \\
\hline
\end{tabular}

\section{Consumo diário por grupo de alimentos e número de refeições ingeridas}

Analisando os dados da Tabela 4, pode-se perceber que os atletas do sexo masculino consomem mais alimentos de todos os grupos, quando comparados com o sexo feminino, exceto para o grupo do leite, onde $90 \%$ das atletas citaram consumir alimentos deste grupo. Outro dado que chama a atenção é o referente ao consumo de vegetais e frutas, somente $30 \%$ dos rapazes e $25 \%$ das moças reportam o consumo deste grupo de alimentos.

No que se refere ao número de refeições ingeridas, verifica-se que os indivíduos de ambos os sexos possuem um hábito de consumir quatro refeições: desjejum ( $80 \%$ dos atletas de ambos os sexos), lanche da manhã ( $87 \%$ do sexo masculino e $80 \%$ do sexo feminino), almoço ( $100 \%$ dos atletas de ambos os sexos) e jantar (100\% dos atletas de ambos os sexos) (Tabela 5).

\section{DISC USSÃO E CONCLUSÃO}

Recentemente foi admitido que durante exercícios intensos de longa duração, quando os estoques de glicogênio são depletados, a proteína é utilizada como fonte energética, pode-se levar a um balanço nitrogenado negativo (LEMON, 1987). Entretanto, quando a ingestão energética e protéica são suficientes, isto não ocorre.

Ao analisar a adequação da dieta encontra-se que a adequação energética está abaixo do recomendado e a adequação protéica está normal para o sexo feminino (Tabela 3). Pode-se supor que as atletas podem estar desenvolvendo um balanço nitrogenado negativo. Quanto aos atletas masculinos, a sua alta ingestão protéica pode estar sendo desviada para fonte energética.

Vários autores também encontraram uma contribuição percentual de lipídeos superior a 
recomendação e citam que os atletas consomem um alto percentual deste nutriente, por acharem que o mesmo, além de fornecer energia mais imediata que os carboidratos, fornecem uma refeição com menor volume (SHORT \& SHORT, 1983; BROTHERHOOD, 1984; GRANDJEAN, 1989; BENSON et al., 1990; FABER et al., 1990).

No que se refere aos carboidratos, sabe-se que estes nutrientes são importantes para os atletas, independente da atividade desportiva que participam. Os atletas deste estudo consomem uma dieta típica de muitos americanos, ou seja, superior ao recomendado em gordura e proteínas e relativamente baixa em carboidratos.

BURKE \& READ (1987), reportam que atletas australianos consomem aproximadamente $60 \%$ do seu total de calorias provenientes dos carboidratos. GREEN et al. (1989), acharam em trabalho com triatletas, uma contribuição percentual de carboidratos maior que $60 \%$. Como pode-se perceber estes dados não são coincidentes neste trabalho, entretanto atendem a recomendação.

CHEN et al. (1989), encontraram um consumo de carboidratos por parte dos nadadores por eles estudados menor que $40 \%$ do total energético. BENSON et al. (1990) encontraram para nadadores do sexo feminino uma percentagem de energia fornecida por carboidratos inferior a 55\% enquanto que VAN ERP BAART et al. (1989), encontraram para o sexo masculino um valor menor que $50 \%$, dados estes que são coincidentes com os nossos.

O conteúdo dos carboidratos na dieta pode ter uma grande influência sobre a sensação de fadiga e sobre o desempenho. Ingestão crônica inadequada destes nutrientes, leva a uma depleção do glicogênio muscular, principalmente com origor dos treinamentos diários intensos e exaustivos (COSTILL et al., 1971; PERNOW \& SALTIN, 1971; COSTILL \& MILLER, 1981).

Vários trabalhos citam a importância do número de refeições por parte de indivíduos atletas. Pesquisas já demonstraram a importância do desjejum para a realização do trabalho. Indivíduos que não fazem esta refeição tem uma queda substancial de seu desempenho em qualquer atividade, principalmente se esta for desenvolvida no período da manhã. De acordo com os nossos resultados, $20 \%$ de nossos atletas não consomem esta refeição, concordando com os dados de vários autores (DAUMK et al., 1950; TUTLLE et al., 1950; MAYER \& BULLEN, 1960) .

A maioria dos autores sugere que os atletas deveriam realizar pelo menos cinco refeições por dia, leves e freqüentes, principalmente em casos de treinamento (MAYER \& BULLEN, 1960; GRANDJEAN, 1989; LEAF \& FRISA, 1989).

Devido ao interesse atual sobre a ingestão e recomendação dietética para atletas, neste trabalho concluiu-se que em relação a ingestão de energia e nutrientes, os atletas de ambos os sexos ingeriam dietas desequilibradas. A ingestão de macronutrientes em termos percentuais apresentou-se elevada para proteína e gordura, e baixa para os carboidratos. Os atletas do sexo masculino obtiveram um percentual de adequação que ultrapassou a recomendação em proteína, lipídeos, vitamina $\mathrm{C}$ e ferro; um percentual normal de adequação para energia e cálcio, e abaixo do recomendado para carboidrato e vitamina A. Os dados mostram também, que os atletas de ambos os sexos não possuem o hábito de consumir frutas e verduras.

O número de refeições ingeridas encontrado foi de quatro (desjejum, lanche, almoço e jantar) ficando abaixo do que se recomenda para atletas, ou seja seis refeições diárias (SHILLS et al., 1995).

\section{REFERÊNCIAS BIBLIOGRÁFICAS}

BAR-OR, O., UNNITHAN, V.B. Nutritional requeriments of young soccer players. Journal of Sports Sciences, London, v.12, p.S39-S42, 1994. Supplement.

BAZARRE, T.L., KLEINER, S.M., LITCH-FORD, M.D. Nutrient intake, body fat and lipid profiles of competitive male and female bodybuilders. Journal of the American College of Nutrition, New York, v.9, n.2, p.136-142,1990.

BELKO, A.Z. Vitamins and exercise: an update. Medicine and Science in Sports and Exercise, Baltimore, v.19, p. S191-S196, 1987. Supplement.

BENSON, J.E., ALLEMANN, Y., THEINTZ, G.E., HOWALD, H. Eating problems and caloric intake, levels in swiss adolescent athletes. International Journal of Sports Medicine, Stuttgart, v.11, n.4, p.249-252,1990.

BLAIR, S.N., SCHOLLER, D.A., CYR, H.N., DIETZ, W. Comparasion of nutrient intake in middle aged men and women runners and controls. Medicine and Science in Sports andExercise, Baltimore, v.13,n.2,p.310-315, 1989. 
BROTHERHOOD, J.R. Nutrition and sports performance. Sports Medicine, New York, v.1, n.1, p.350-389, 1984.

BURKE, L.M., READ, R.S.D. Diet patterns of elite Australians male triathletes Physiologic Sports Medicine, New York, v.15, n.3, p.140-155, 1987.

BUSKIRK, E.R. Ejercicio. In: CONOCIMENTOS actuales sobre nutrición. 6.ed. Washington DC : OPAS, 1991. p.394-402.

BUTTERFIELD, G.E., CALLOWAY, D.H. Physical activity improves protein utilization in young men. British Journal of Nutrition, Cambridge, v.51, n.5, p.171-184, 1984.

CELEJOWA, I., HOMA, M. Food intake, nitrogen and energy balance in weigthlefters during a training camp. Nutrition Metabolism, Massachusets, v.12, n.5, p.259-274, 1970.

CLEMENT, D.B., ASMUNDSON, R.C. Nutritional intake and hematological parameters in the endurance runners. Physiologic Sports Medicine, New York, v.10, n.13, p.232-238, 1982.

CHEN, J.D. Nutritional problems and measures in elite and amateurs athletes. The American Journal of Clinical Nutrition, Bethesda, v.49, n.6, p.1084-1089, 1989.

COSTILL, D.L., MILLER, J.M. Nutrition for endurance sports: carbohydrate and fluids balance. International Journal of Sports Medicine, Stuttgart, v.51, n.1, p.2$14,1981$.

COSTILL, D.L., DOYLE, J.A., STRAUSS, R., LAMB, R. Muscle glycogen utilization during prolonged exercise on sucessives days. Journal of Applied Physiology, Bethesda, v.31, n.6, p.834-838, 1971.

DAUMK, K., TUTTLE, W.W., MARTIN, C., MYERS, L. Effect of various types of breakfast on physiologic response. Journal of the American Dietetic Association, Chicago, v.26, n.3, p.503-506, 1950.

FABER, M., SPINNLER-BENADE, A.J., DAUBTZER, A. Dietary intake, anthropometric measurements and plasma lipid levels in throwing field athletes. International Journal of Sports Medicine, Stuttgart, v.10, n.2, p.140-145, 1990.

GERSOVITZ, M., MADDEN, J.P., WRILGTH, H.S. Validity of the $24 \mathrm{hr}$ dietary recall and seven days record for group comparisions. Journal of the American Dietetic Association, Chicago, v.73, n.7, p.48-55, 1978.

GRANDJEAN, A.C. Macronutrient intake of VS athletes compared with the general population and recomendations made for athletes. The American Journal of Clinical Nutrition, Bethesda, v.49, n.6,p.1070-1076, 1989.
GREEN, D. R., GIBBONS, C., O’TOOLE, M., HILLER, W. An evolution of dietary intakes of triathletes: are RDAS being met? Journal of the American Dietetic Association, Chicago, v.89, n.11, p.430-433, 1989.

HARGREAVES, M. Carbohydrate and lipid requerimentes of soccer. Journal of Sports Sciences, London, v.12, S13-S16, 1994.

HULTMAN, E., THOMSON, J.A., HARRIS, R.C. Work and exercise In: Modern nutrition in health and disease. 17.ed. Phyladelphia : Lea \& Febiger, 1988. p.1001-1022.

HULTMAN, E. Nutritional effects on work performance. American Journal of Clinical Nutrition, Bethesda, v.49, n.6,p.949-957, 1989.

INSTITUTO BRASILEIRO DE GEOGRAFIA E ESTATÍSTICA. Estudo Nacional de Despesa Familiar (ENDEF): tabela de composição de alimentos. Rio de Janeiro, 1979.v.3.

KHOO, C. S., BURGESS, M.L., NORRIS, J. M., DAVIS, J.M. Nutrition intake and eating habits of triathletes. Annals Sports Medicine, Boston, v.3, p.144-150, 1987.

KRALL, E.A., DWYER, J.T. Validity of a food frequency questionaire and a food diary in a short-term recall situations. Journal of the American Dietetic Association, Chicago, v.73, n.2, p.48-55, 1987.

LEAF, A. What constitutes good nutrition for the athlete and pos athletes? American Journal of Clinical Nutrition, Bethesda, v.49, n.6, p.936-937, 1989.

LEAF, A., FRISA, K.B. Eating for health or for athletic performance. American Journal of Clinical Nutrition, Bethesda, v.49, n.6, p.1066-1069, 1989.

LEMON, P.W.R. Protein and exercise update. Medicine and Science in Sports and Exercise. Baltimore, v.19, p.S179-S190, 1987. Supplement.

LEMON, P.W.R. Protein requeriment of soccer. Journal of Sports Science, London, v.12, p.S17-S22, 1994.

LEMON, P.W.R., YARASHESKI, K.E., DOLONY, D.G. The importance of protein for athletes. Sports Medicine, Boston, v.1, n.5, p.474-480, 1984.

MAYER, J., BULLEN, B. Nutrition for athletic performance. The American Physiologic Society, Boston, v.40, n.3, p.369-397, 1960.

MONSEN, E.R. Recommended Dietary Allowances (RDA's) 10.ed. Journal of the American Dietetic Association,. Chicago, v.89, n.12,p.1748-1752, 1989.

ORGANIZACIÓN MUNDIAL DE LA SALUD. Necessidades de energia y de proteinas. Genebra, 1985.p.220.(Informe Técnico, n.724). 
PERNOW, B., SALTIN, B. Availability of substrates and capacity for prolonged heavy exercise in man. Journal of Applied Physiology, Bethesda, v.31,n.6, p.416-422, 1971.

SOARES, E.A., MIDORI, I., BURINI, R.C. Estudo antropométrico e dietético de nadadores competitivos de áreas metopolitanas da Região Sudeste do Brasil. Revista de Saúde Pública, São Paulo, v.28, n.1, p.9-19, 1994.

SHILLS, M.E., OLSON, J.A., SHIKE, M. Modern nutrition in health and disease. 8.ed. London : Lea \& Feiber, 1995.p.623.

SHORT, S.H., SHORT, W.R. Four years study of university athletes dietary intake. Journal of the American Dietetic Association, Chicago, v.82, n.6, p.32-45, 1983.

TUTTLE, W.W., DAUM, K., MYERS, L., MARTIN, C. Effect of ommitting breakfast on physiologic response of men. Journal of the American Dietetic Association, Chicago, v.26, n.7, p.330-338, 1950.
VAN ERP-BAART, A.M.J., SARIS, W.H.M., BIENKHORST, R.A., ELVERS, J.W.H. Nationwide survey on nutritional habits in elite athletes. Part I: Energy, carbohydrate, protein and fat intake. International Journal of Sports Medicine, Stuttgart, v.10, p.503-510, 1989. Supplement.

VANNUCCHI, H., MENEZES, E.W., CAMPANA, A.D., LAJOLO, F. M. Aplicações das recomendações nutricionais adaptadas à população brasileira. Sociedade Brasileira de Alimentação e Nutrição, São Paulo, v.2, p.155, 1990.

WILLIAMS, C., DEVLIN, J. Foods, nutrition and sports performance. London : E \& FN SPON, 1994. p.194. (International Concensus Held 4 - 6 Febuary 1991).

Recebidoparaapublicaçãoem 11 demarçoeaceitoem 11 deagosto de 1997. 